\title{
Braneworld tensor anisotropies in the CMB
}

\author{
Bernard Leong, ${ }^{1, *}$ Anthony Challinor, ${ }^{1, \dagger}$ Roy Maartens, ${ }^{2,}{ }^{\ddagger}$ and Anthony Lasenby ${ }^{1, \S}$ \\ ${ }^{1}$ Astrophysics Group, Cavendish Laboratory, Madingley Road, Cambridge CBз OHE, UK \\ ${ }^{2}$ Institute of Cosmology $\&$ Gravitation, Portsmouth University, Portsmouth PO1 2EG, UK
}

(Dated: February 2, 2008)

\begin{abstract}
Cosmic microwave background (CMB) observations provide in principle a high-precision test of models which are motivated by $\mathrm{M}$ theory. We set out the framework of a program to compute the tensor anisotropies in the CMB that are generated in braneworld models. In the simplest approximation, we show the braneworld imprint as a correction to the power spectra for standard temperature and polarization anisotropies.
\end{abstract}

PACS numbers: $04.50 .+\mathrm{h}, 98.80 . \mathrm{Cq}$

\section{INTRODUCTION}

The early universe provides a testing ground for theories of gravity. The standard cosmological model, based on general relativity with an inflationary era, is very effective in accounting for a broad range of observed features of the universe. However, the lack of a consistent theoretical framework for inflation, together with the ongoing puzzles on the nature of dark matter and dark energy, indicate that cosmology may be probing the limits of validity of general relativity.

M theory is considered to be a promising potential path to quantum gravity. As such, it is an important candidate for cosmological testing. In the absence of a sufficiently general M-theoretic model of cosmology, we can use phenomenological models that share some of the key features of $\mathrm{M}$ theory, including branes. In brane cosmology, the observable universe is a 1+3-dimensional "brane" surface moving in a higher-dimensional "bulk" spacetime. Standardmodel fields are confined to the brane, while gravity propagates in the bulk. The simplest, and yet sufficiently general, phenomenological braneworld models are those based on the Randall-Sundrum II scenario [1]. These models have the additional advantage that they provide a framework for investigating aspects of holography and the AdS/CFT correspondence.

In the generalized RSII models, analyzed via an elegant geometrical approach in Ref. [2], the bulk is a 1+4dimensional spacetime, with non-compact extra spatial dimension. What prevents gravity from 'leaking' into the extra dimension at low energies is the negative bulk cosmological constant $\Lambda_{5}=-6 / \ell^{2}$, where $\ell$ is a curvature scale of the bulk. In the weak-field static limit, null results in tests for deviations from Newton's law impose the limit $\ell \lesssim 1 \mathrm{~mm}$. The negative $\Lambda_{5}$ is offset by the positive brane tension $\lambda$, which defines the energy scale dividing low from high energies. The limit $\ell<1 \mathrm{~mm}$ implies $\lambda>(100 \mathrm{GeV})^{4}$, and the effective cosmological constant on the brane is

$$
\Lambda=\frac{1}{2}\left(\Lambda_{5}+\kappa^{2} \lambda\right),
$$

where $\kappa^{2}=8 \pi G=8 \pi / M_{4}^{2}$, and $M_{4} \sim 10^{19} \mathrm{GeV}$ is the effective Planck scale on the brane. A further intriguing feature of the braneworld scenario is that, because of the large extra dimensions, the fundamental energy scale of gravity can be dramatically lower than the effective Planck scale on the brane - as low as $\sim \mathrm{TeV}$ in some scenarios. In generalized RSII models, the fundamental scale is higher, $M_{5}>10^{5} \mathrm{TeV}$, and is related to $M_{4}$ via $M_{5}^{3}=M_{4}^{2} / \ell$.

At energies well above the brane tension $\lambda$, gravity becomes 5 -dimensional and significant corrections to general relativity occur. There are also corrections that can operate at low energies, mediated by bulk graviton or Kaluza-Klein (KK) modes. Both types of correction play an important role in tensor perturbations.

The background cosmological dynamics of a Friedmann brane in Schwarzschild-Anti de Sitter (AdS) bulk are well understood [3], including the high-energy modifications to inflation [4]. High-energy inflation on the brane generates a zero-mode (4D graviton mode) of tensor perturbations, and stretches it to super-Hubble scales. This zero-mode has

\footnotetext{
*Electronic address: cwbl2@mrao.cam.ac.uk

$\dagger$ Electronic address: a.d.challinor@mrao.cam.ac.uk

$\ddagger$ Electronic address: roy.maartens@port.ac.uk

$\S$ Electronic address: a.n.lasenby@mrao.cam.ac.uk
} 
the same qualitative features as in general relativity, remaining frozen at constant amplitude while beyond the Hubble horizon, but the overall amplitude is higher [5]. The massive KK modes (5D graviton modes) remain in the vacuum state during slow-roll inflation. The evolution of the super-Hubble zero mode is the same as in general relativity, so that high-energy braneworld effects in the early universe serve only to re-scale the amplitude. However, when the zero mode re-enters the Hubble horizon, massive KK modes can be excited. Qualitative arguments [6, 7] indicate that this is a very small effect, but it remains to be properly quantified, so that the signature on the CMB may be calculated, and constraints may be imposed on the braneworld parameters.

We develop here a formalism to compute the tensor anisotropies in the CMB, which incorporates the early-universe high-energy braneworld effects, and we carefully delineate what is known on the brane from what is required from bulk equations. Once the 5D solutions are provided, our formalism, with its modified CMB code (based on CAMB [8, 9]), is able to compute these anisotropies. We illustrate this by using a simple approximation to the 5D effects (cf. the analysis of the braneworld scalar Sachs-Wolfe effect in Ref. [10]).

\section{BRANEWORLD DYNAMICS AND TENSOR PERTURBATIONS}

There has been an explosion of interest in the theory of cosmological perturbations in braneworlds and their implications for observational cosmology (see e.g. Refs. [11]).

In this paper, we follow the same general formalism previously developed to analyze braneworld scalar anisotropies in the CMB [12]. This is based on the 1+3-covariant perturbation theory for CMB anisotropies [13], generalized to incorporate braneworld effects. (See Ref. [12] for further details and definitions.)

The field equations induced on the brane are

$$
G_{a b}=-\Lambda g_{a b}+\kappa^{2} T_{a b}+6 \frac{\kappa^{2}}{\lambda} \mathcal{S}_{a b}-\mathcal{E}_{a b}
$$

where $\mathcal{S}_{a b} \sim\left(T_{a b}\right)^{2}$ carries high-energy corrections, and is negligible for matter energy densities $\rho \ll \lambda$, while $\mathcal{E}_{a b}$, the projection of the bulk Weyl tensor on the brane, carries corrections from KK or $5 \mathrm{D}$ graviton effects. From the brane-observer viewpoint, the energy-momentum corrections in $\mathcal{S}_{a b}$ are local, whereas the KK corrections in $\mathcal{E}_{a b}$ are nonlocal $[15,16]$, since they incorporate 5D gravity wave modes. These nonlocal corrections to the Einstein equations on the brane cannot be determined purely from data on the brane, and so the induced field equations are not a closed system; one needs to supplement them by $5 \mathrm{D}$ equations governing $\mathcal{E}_{a b}$.

The trace free $\mathcal{E}_{a b}$ contributes an effective energy density $\rho^{*}$, pressure $\rho^{*} / 3$, momentum density $q_{a}^{*}$ and anisotropic stress $\pi_{a b}^{*}$ on the brane, which collectively incorporate the spin- 0 , spin- 1 and spin- 2 modes of the $5 \mathrm{D}$ graviton, and through which the brane "feels" the bulk gravitational field. Then the braneworld corrections can conveniently be consolidated into an effective total energy density, pressure, momentum density and anisotropic stress:

$$
\begin{aligned}
\rho^{\mathrm{eff}} & =\rho\left(1+\frac{\rho}{2 \lambda}+\frac{\rho^{*}}{\rho}\right) \\
P^{\mathrm{eff}} & =P+\frac{\rho}{2 \lambda}(2 P+\rho)+\frac{\rho^{*}}{3}, \\
q_{a}^{\mathrm{eff}} & =q_{a}\left(1+\frac{\rho}{\lambda}\right)+q_{a}^{*} \\
\pi_{a b}^{\mathrm{eff}} & =\pi_{a b}\left(1-\frac{\rho+3 P}{2 \lambda}\right)+\pi_{a b}^{*},
\end{aligned}
$$

where $\rho$ and $P$ are the total matter density and pressure, $q_{a}$ is the total matter momentum density, and $\pi_{a b}$ is the matter anisotropic stress. We have neglected terms quadratic in $q_{a}$ and $\pi_{a b}$ since these do not contribute to the fluctuations in the effective variables in linear perturbation theory.

In the background, $q_{a}^{*}=0=\pi_{a b}^{*}$. The modified Friedmann equations are

$$
\begin{aligned}
H^{2} & =\frac{\kappa^{2}}{3} \rho^{\mathrm{eff}}+\frac{1}{3} \Lambda-\frac{K}{a^{2}}, \\
\dot{H} & =-\frac{\kappa^{2}}{2}\left(\rho^{\mathrm{eff}}+P^{\mathrm{eff}}\right)+\frac{K}{a^{2}},
\end{aligned}
$$

and the KK energy density behaves like 'dark' radiation:

$$
\rho^{*} \propto \frac{1}{a^{4}} .
$$


The $(1+3)$-covariant description of tensor perturbations in general relativity $[13,14]$ generalizes naturally to braneworld cosmology [15]. Tensor modes on the brane are characterized by the transverse traceless shear $\sigma_{a b}$, which is a tensor potential for the electric and magnetic Weyl tensors $E_{a b}$ and $H_{a b}$. The sources for linearized tensor modes are the matter anisotropic stress $\pi_{a b}$ and the KK anisotropic stress $\pi_{a b}^{*}$. The Boltzmann equation governs $\pi_{a b}$. As pointed out above, the KK anisotropic stress, which carries the imprint of $5 \mathrm{D}$ gravitational wave modes on the brane, is not determined by $4 \mathrm{D}$ equations on the brane. For the moment, we assume that $\pi_{a b}^{*}$ is given from a solution to the $5 \mathrm{D}$ equations. Below we will discuss what this entails and how one can approximate the solution.

The transverse traceless quantities can be expanded in electric $\left(Q_{a b}^{(k)}\right)$ and magnetic $\left(\bar{Q}_{a b}^{(k)}\right)$ parity tensor harmonics [13], with dimensionless coefficients:

$$
\begin{aligned}
E_{a b} & =\sum_{k}\left(\frac{k}{a}\right)^{2}\left[E_{k} Q_{a b}^{(k)}+\bar{E}_{k} \bar{Q}_{a b}^{(k)}\right] \\
H_{a b} & =\sum_{k}\left(\frac{k}{a}\right)^{2}\left[H_{k} Q_{a b}^{(k)}+\bar{H}_{k} \bar{Q}_{a b}^{(k)}\right] \\
\sigma_{a b} & =\sum_{k} \frac{k}{a}\left[\sigma_{k} Q_{a b}^{(k)}+\bar{\sigma}_{k} \bar{Q}_{a b}^{(k)}\right] \\
\pi_{a b} & =\rho \sum_{k}\left[\pi_{k} Q_{a b}^{(k)}+\bar{\pi}_{k} \bar{Q}_{a b}^{(k)}\right] \\
\pi_{a b}^{*} & =\rho \sum_{k}\left[\pi_{k}^{*} Q_{a b}^{(k)}+\bar{\pi}_{k}^{*} \bar{Q}_{a b}^{(k)}\right] .
\end{aligned}
$$

Using $H_{a b}=\operatorname{curl} \sigma_{a b}$, we arrive at the coupled equations

$$
\begin{aligned}
& \frac{k}{a^{2}}\left(\sigma_{k}^{\prime}+\mathcal{H} \sigma_{k}\right)+\frac{k^{2}}{a^{2}} E_{k}-\frac{\kappa^{2}}{2} \rho \pi_{k}=\kappa^{2}(2-3 \gamma) \frac{\rho^{2}}{4 \lambda} \pi_{k}+\frac{\kappa^{2}}{2} \rho \pi_{k}^{*}, \\
& \frac{k^{2}}{a^{2}}\left(E_{k}^{\prime}+\mathcal{H} E_{k}\right)-k\left(\frac{k^{2}}{a^{2}}+\frac{3 K}{a^{2}}-\frac{\kappa^{2}}{2} \gamma \rho\right) \sigma_{k}+\frac{\kappa^{2}}{2} \rho \pi_{k}^{\prime}-\frac{\kappa^{2}}{2}(3 \gamma-1) \mathcal{H} \rho \pi_{k} \\
& =-\frac{\kappa^{2}}{4 \lambda}\left\{2 k \gamma \rho^{2} \sigma_{k}-(3 \gamma-2) \rho^{2} \pi_{k}^{\prime}-\left[3 \gamma^{\prime}-(3 \gamma-2)(6 \gamma-1) \mathcal{H}\right] \rho^{2} \pi_{k}\right\} \\
& \quad-\frac{2}{3} k \kappa^{2} \rho^{*} \sigma_{k}-\frac{\kappa^{2}}{2}\left[\rho \pi_{k}^{*^{\prime}}+(1-3 \gamma) \mathcal{H} \rho \pi_{k}^{*}\right]
\end{aligned}
$$

where a prime denotes $d / d \tau$, with $\tau$ conformal time, $\mathcal{H}=a^{\prime} / a$, and the (non-constant) parameter $\gamma$ is defined by $P=(\gamma-1) \rho$. Equations (15) and (16), with all braneworld terms on the right-hand sides, determine the tensor anisotropies in the CMB, once $\pi_{k}$ and $\pi_{k}^{*}$ are given. The former is determined by the Boltzmann equation in the usual way [13], except that the background dynamics are altered by braneworld effects. The latter requires a solution of the 5D perturbation equations.

The solution for the non-local anisotropic stress will be of the form

$$
\left.\pi_{k}^{*}(\tau) \propto \int d \tilde{\tau} \mathcal{G}(\tau, \tilde{\tau}) F\left[\pi_{k}, \sigma_{k}\right]\right|_{\tilde{\tau}}
$$

where $\mathcal{G}$ is a retarded Green's function evaluated on the brane. The functional $F$ is known in the case of a Minkowski background [17], but not in the cosmological case. (An equivalent integro-differential formulation of the problem is given in Ref. [18]; see also Ref. [19].) Once $\mathcal{G}$ and $F$ are determined, Eq. (17) can in principle be incorporated into a modified version of Boltzmann codes such as CAMB [8] or CMBFAST [20]. It remains a major task of braneworld cosmological perturbation theory to find this solution, or its equivalent forms in other formalisms. In the meanwhile, in order to make progress towards understanding braneworld signatures on tensor CMB anisotropies, we can consider approximations to the solution.

The nonlocal nature of $\pi_{k}^{*}$, as reflected in Eq. (17), is fundamental, but is also the source of the great complexity of the problem. The lowest level approximation to $\pi_{k}^{*}$ is local. Despite removing the key aspect of the KK anisotropic stress, we can get a feel for its influence on the CMB if we capture at least part of its qualitative properties. The key qualitative feature is that inhomogeneity and anisotropy on the brane are a source for KK modes in the bulk which "backreact" [15] or "feed back" [16], onto the brane. The transverse traceless part of inhomogeneity and anisotropy on the brane is given by the transverse traceless anisotropic stresses in the geometry, i.e. by the matter anisotropic 
stress $\pi_{a b}$ and the shear anisotropy $\sigma_{a b}$. The radiation and neutrino anisotropic stresses are in turn sourced by the shear to lowest order (neglecting the role of the octupole and higher Legendre moments).

Thus the simplest local approximation which reflects the essential qualitative feature of the spin- 2 KK modes is

$$
\kappa^{2} \pi_{a b}^{*}=-\zeta H \sigma_{a b}, \quad \zeta^{\prime}=0
$$

where $\zeta$ is a dimensionless KK parameter, with $\zeta=0$ corresponding to no KK effects on the brane, and $\zeta=0=\lambda^{-1}$ giving the general relativity limit. [Note that for tensor perturbations, where there is no freedom over the choice of frame (i.e. $u^{a}$ ), there is no gauge ambiguity in Eq. (18). However, for scalar or vector perturbations, this relation could only hold in one frame, since $\pi_{a b}^{*}$ is frame-invariant in linear theory while $\sigma_{a b}$ is not.]

The approximation in Eq. (18) has the qualitative form of a shear viscosity, which suggests that KK effects lead to a damping of tensor anisotropies. This is indeed consistent with the conversion of part of the zero-mode at Hubble reentry into massive KK modes [5, 7]. The conversion may be understood equivalently as the emission of KK gravitons into the bulk, and leads to a loss of energy in the $4 \mathrm{D}$ graviton modes on the brane, i.e. to an effective damping. The approximation in Eq. (18) therefore also incorporates this key feature qualitatively.

With this first approximation, we can close the system of equations on the brane by adding the equation

$$
\kappa^{2} \rho \pi_{k}^{*}=-\zeta \mathcal{H} \frac{k}{a^{2}} \sigma_{k}
$$

We will also assume $K=0=\rho^{*}$ in the background. The parameter $\zeta$ (together with the brane tension $\lambda$ ) then controls braneworld effects on the tensor CMB anisotropies in this simplest approximation.

\section{CMB TENSOR POWER SPECTRA}

Ignoring the photon anisotropic stress (i.e. $\pi_{a b}=0$ ), the variable $u_{k} \equiv a^{1+\zeta / 2} \sigma_{k}$ satisfies the equation of motion

$$
u_{k}^{\prime \prime}+\left[k^{2}+2 K-\frac{\left(a^{-1-\zeta / 2}\right)^{\prime \prime}}{a^{-1-\zeta / 2}}\right] u_{k}=0,
$$

where we have used Eq. (18). In flat models $(K=0)$ on large scales there is a decaying solution $\sigma_{k} \propto a^{-(2+\zeta)}$. Since Eq. (20) contains no first derivative term the Wronskian is conserved. On large scales we can use the solution $\sigma_{k} \propto a^{-(2+\zeta)}$ to write the conserved Wronskian as $W=\sigma_{k}^{\prime}+(2+\zeta) \mathcal{H} \sigma_{k}$. (The Wronskian vanishes in the decaying mode.) Integrating gives the following two independent solutions on large scales in flat models:

$$
\sigma_{k}=\left\{\begin{array}{l}
A_{k} a^{-(2+\zeta)} \\
B_{k} a^{-(2+\zeta)}
\end{array} \int^{\tau} d \tilde{\tau} a(\tilde{\tau})^{2+\zeta},\right.
$$

where $A_{k}$ and $B_{k}$ are constants of integration. If we let $\zeta \rightarrow 0$, we recover the results in Ref. [9] for the general relativity case.

The conserved Wronskian is proportional to the metric perturbation variable, $H_{T}$, characterising the amplitude of $4 \mathrm{D}$ gravitational waves. In flat models $H_{T}$ is related to the covariant variables quite generally by

$$
H_{T k}=\frac{\sigma_{k}^{\prime}}{k}+2 E_{k} .
$$

Ignoring photon anisotropic stress, we can eliminate the electric part of the Weyl tensor via the shear propagation equation (15) to find $k H_{T k}=-\sigma_{k}^{\prime}-(\zeta+2) \mathcal{H} \sigma_{k}=-W$. The fact that $H_{T}$ is conserved on large scales in flat models in the absence of photon anisotropic stress can also be seen directly from its propagation equation,

$$
H_{T k}^{\prime \prime}+(2+\zeta) \mathcal{H} H_{T k}^{\prime}+k^{2} H_{T k}=0
$$

We can solve Eq. (20) on all scales in the high-energy $\left(\rho \gg \lambda\right.$ and $\left.a \propto \tau^{1 / 3}\right)$ and low-energy $(\rho \ll \lambda$ and $a \propto \tau)$ radiation-dominated regimes, and during matter-domination $\left(a \propto \tau^{2}\right)$. The solutions are

$$
\begin{aligned}
& u_{k}(\tau)=\sqrt{k \tau}\left[c_{1} J_{\frac{1}{6}(5+\zeta)}(k \tau)+c_{2} Y_{\frac{1}{6}(5+\zeta)}(k \tau)\right] \quad \text { (high energy radiation), } \\
& u_{k}(\tau)=\sqrt{k \tau}\left[c_{3} J_{\frac{1}{2}(3+\zeta)}(k \tau)+c_{4} Y_{\frac{1}{2}(3+\zeta)}(k \tau)\right] \quad \text { (low energy radiation), } \\
& u_{k}(\tau)=\sqrt{k \tau}\left[c_{5} J_{\frac{5}{2}+\zeta}(k \tau)+c_{6} Y_{\frac{5}{2}+\zeta}(k \tau)\right] \quad \text { (matter domination), }
\end{aligned}
$$


where $c_{i}$ are integration constants. The solutions for the electric part of the Weyl tensor can be found from Eq. (15). For modes of cosmological interest the wavelength is well outside the Hubble radius at the transition from the highenergy regime to the low-energy. It follows that the regular solution (labelled by $c_{1}$ ) in the high-energy regime will only excite the regular solution $\left(c_{3}\right)$ in the low-energy, radiation-dominated era. Performing a series expansion, we arrive at the appropriate initial conditions for large-scale modes in the low-energy radiation era:

$$
\begin{aligned}
H_{T k} & =1-\frac{(k \tau)^{2}}{2(3+\zeta)}+\frac{(k \tau)^{4}}{8(3+\zeta)(5+\zeta)}+O\left[(k \tau)^{6}\right], \\
\sigma_{k} & =-\frac{k \tau}{3+\zeta}+\frac{k^{3} \tau^{3}}{2(3+\zeta)(5+\zeta)}+O\left[(k \tau)^{5}\right] \\
E_{k} & =\frac{(4+\zeta)}{2(3+\zeta)}-\frac{(k \tau)^{2}(8+\zeta)}{4(3+\zeta)(5+\zeta)}+O\left[(k \tau)^{4}\right] .
\end{aligned}
$$

In the limit $\zeta \rightarrow 0$, we recover the general relativity results [13].

For modes that are super-Hubble at matter-radiation equality (i.e. $k \tau_{\text {eq }} \ll 1$ ), the above solution joins smoothly onto the regular solution labelled by $c_{5}$ in Eq. (26). For $k \tau_{\text {eq }} \gg 1$, the shear during matter domination takes the form

$$
\sigma_{k}=-2^{\frac{3}{2}+\zeta} \Gamma\left(\frac{5}{2}+\zeta\right)(k \tau)^{-\left(\frac{3}{2}+\zeta\right)} J_{\frac{5}{2}+\zeta}(k \tau) .
$$

In the opposite limit, the wavelength is well inside the Hubble radius at matter-radiation equality. The asymptotic form of the shear in matter domination is then

$$
\sigma_{k} \sim \frac{\Gamma\left[\frac{1}{2}(3+\zeta)\right]}{\sqrt{\pi}}\left(\frac{2 \tau_{\mathrm{eq}}}{\tau}\right)^{1+\zeta / 2}(k \tau)^{-(1+\zeta / 2)} \sin (k \tau-\pi \zeta / 4) .
$$

We use the initial conditions, Eqs. (27)-(29), in a modified version of the CAMB code to obtain the tensor temperature and polarization power spectra. The temperature and electric polarization spectra are shown in Figs. 1 and 2 for a scale-invariant initial power spectrum. The normalisation is set by the initial power in the gravity wave background. Figs 1 and 2, together with Eqs. (24)-(29), are the main result of this work, and we now discuss the physical conclusions following from these results.

\section{DISCUSSION}

As expected, we find that the power spectra are insensitive to high-energy effects, i.e. effectively independent of the brane tension $\lambda$ : the $\zeta=0$ curve in Fig. 1 is indistinguishable from that of the general relativity model (both power spectra are identical at the resolution of the plot). For the computations, we have used the lowest value of the brane tension $\lambda=(100 \mathrm{GeV})^{4}$, consistent with the tests of Newton's law.

There are three notable effects visible in Figs. 1 and 2, arising as the physical consequences of our approximate model of the KK stress: (i) the power on large scales reduces with increasing KK parameter $\zeta$; (ii) features in the spectrum shift to smaller angular scales with increasing $\zeta$; and (iii) the power falls off more rapidly on small scales as $\zeta$ increases. Neglecting scattering effects, the shear is the only source of linear tensor anisotropies (see e.g. Ref. [13]). For $1 \ll l<60$ the dominant modes to contribute to the temperature $C_{l}$ s are those whose wavelengths subtend an angle $\sim 1 / l$ when the shear first peaks (around the time of Hubble crossing). The small suppression in the $C_{l}$ s on large scales with increasing $\zeta$ arises from the reduction in the peak amplitude of the shear at Hubble entry [see Eq. (30)], qualitatively interpreted as the loss of energy in the $4 \mathrm{D}$ graviton modes to $5 \mathrm{D}$ KK modes.

Increasing $\zeta$ also has the effect of adding a small positive phase shift to the oscillations in the shear on sub-Hubble scales, as shown e.g. by Eq. (31). The delay in the time at which the shear first peaks leads to a small increase in the maximum $l$ for which $l(l+1) C_{l}$ is approximately constant, as is apparent in Fig. 1 . The phase shift of the subsequent peaks in the shear has the effect of shifting the peaks in the tensor $C_{l}$ s to the right. For $l>60$ the main contribution to the tensor anisotropies at a given scale is localized near last scattering and comes from modes with wavenumber $k \sim l / \tau_{0}$, where $\tau_{0}$ is the present conformal time. On these scales the gravity waves have already entered the Hubble radius at last scattering. Such modes are undergoing adiabatic damping by the expansion and this results in the sharp decrease in the anisotropies on small scales. Increasing the KK parameter $\zeta$ effectively produces more adiabatic damping and hence a sharper fall off of power. The transition to a slower fall off in the $C_{l} \mathrm{~s}$ at $l \sim 200$ is due to the weaker dependence of the amplitude of the shear on wavenumber at last scattering for modes that have entered the Hubble radius during radiation domination [21]. [The asymptotic expansion of Eq. (30) gives the shear 


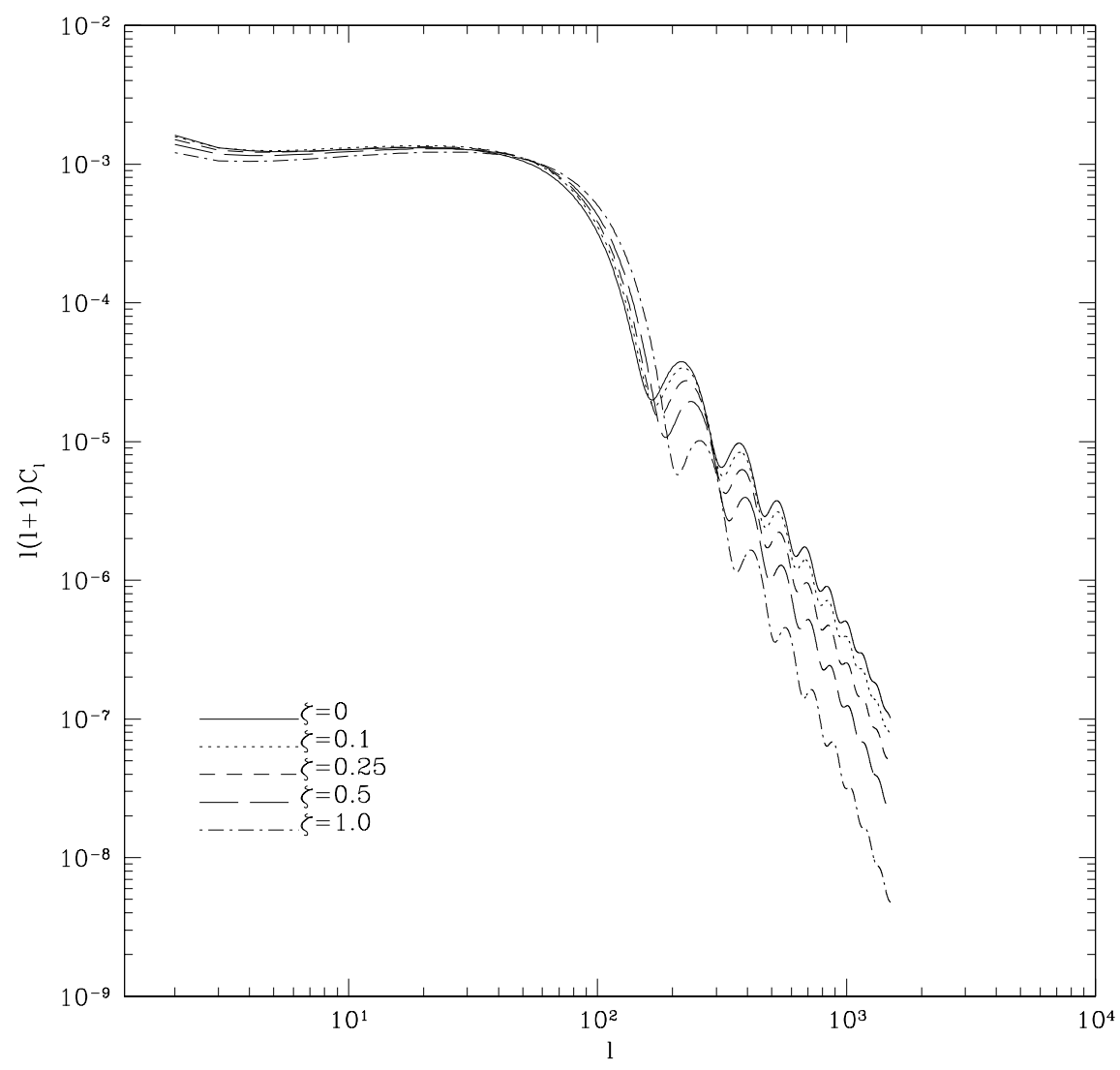

FIG. 1: The temperature power spectrum for tensor perturbations in braneworld models using the approximation in Eq. (18), with $\zeta$ the dimensionless KK parameter. Models are shown with $\zeta=0.0,0.1,0.25,0.5$ and 1.0. The initial tensor power spectrum is scale invariant and we have adopted an absolute normalisation to the power in the primordial gravity wave background. The background cosmology is the spatially flat $\Lambda$ CDM (concordance) model with density parameters $\Omega_{b}=0.035$, $\Omega_{c}=0.315, \Omega_{\Lambda}=0.65$, no massive neutrinos, and the Hubble constant $H_{0}=65 \mathrm{~km} \mathrm{~s}^{-1} \mathrm{Mpc}^{-1}$.

amplitude $\propto k^{-(2+\zeta)}$ at fixed $\tau$, whereas for modes that were sub-Hubble at matter-radiation equality Eq. (31) gives the amplitude $\propto k^{-(1+\zeta / 2)}$.]

Similar comments apply to the tensor electric polarization $C_{l}^{E}$, shown in Fig. 2. As with the temperature anisotropies, we see the same shifting of features to the right and increase in damping on small scales. Since polarization is only generated at last scattering (except for the feature at very low $l$ that arises from scattering at reionization, with an assumed optical depth $\tau_{C}=0.03$ ), the large-scale polarization is suppressed, since the shear (and hence the temperature quadrupole at last scattering) is small for super-Hubble modes. In matter domination the large-scale shear is $\sigma_{k}=-k \tau /(5+2 \zeta)$; the reduction in the magnitude of the shear with increasing KK parameter $\zeta$ is clearly visible in the large-angle polarization. The braneworld modification to the tensor magnetic polarization $C_{l}^{B}$ has the same qualitative features as in the electric case.

In principle, observations can constrain the $\mathrm{KK}$ parameter $\zeta$, which controls the generation of 5D modes within our simplified local approximation, Eq. (18). The other braneworld parameter $\lambda$, the brane tension, is not constrained within our approximation. In practice, the tensor power spectra have not been measured, and the prospect of useful data is still some way off. What is more important is the theoretical task of improving on the simplified local approximation we have introduced. This approximation has allowed us to encode aspects of the qualitative features of braneworld tensor anisotropies, which we expect to survive in modified form within more realistic approximations. However, a proper understanding of braneworld effects must incorporate the nonlocal nature of the KK graviton modes, as reflected in the general form of Eq. (17). It is also necessary to investigate the scalar anisotropies, which have a dominant contribution to the measured power spectra. These may reveal new braneworld imprints that are more amenable to observational testing. 


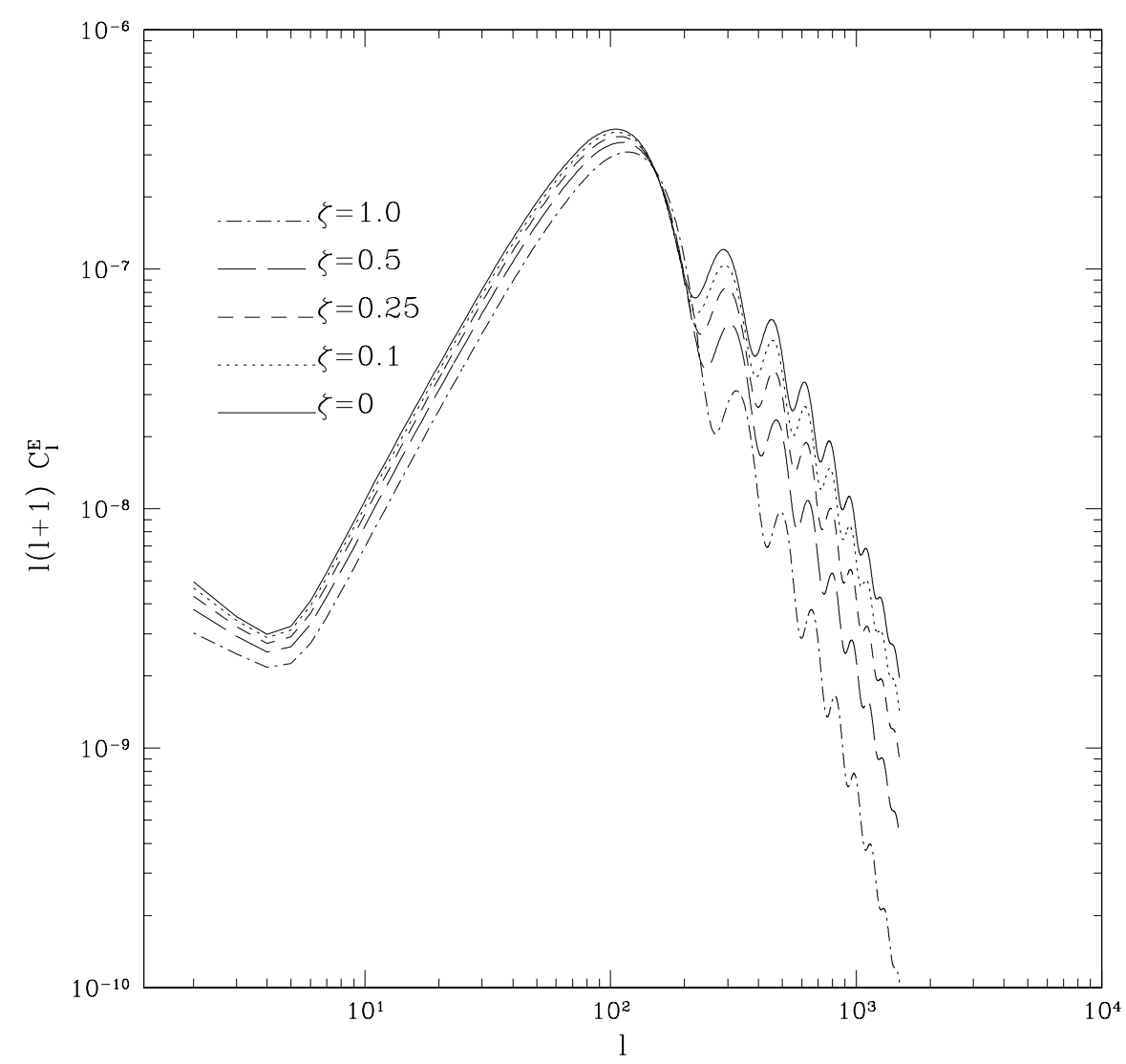

FIG. 2: The electric polarization power spectrum for tensor perturbations for the same braneworld models as in Fig. 1.

\section{Acknowledgments}

B.L. thanks the Universities of Cape Town and Portsmouth for hospitality, during which part of the work was done. B.L. thanks Y.L. Loh, A. Lewis, A. Kahle, G. Chon and J. Weller for assistance with various computational matters, P. Dunsby and C. van de Bruck for helpful and critical discussions. B.L. is supported by an Overseas Research Studentship, the Cambridge Commonwealth Trust and the Lee Foundation, Singapore. A.C. acknowledges a PPARC Postdoctoral Fellowship.

[1] L. Randall and R. Sundrum, Phys. Rev. Lett. 83, 4690 (1999).

[2] T. Shiromizu, K. Maeda, and M. Sasaki, Phys. Rev. D 62, 024012 (2000).

[3] P. Binetruy C. Deffayet U. Ellwanger, and D. Langlois, Phys. Lett. B 477, 285 (2000).

[4] R. Maartens, D. Wands, B. Bassett, and I. Heard, Phys. Rev. D 62, 041301 (2000).

[5] D. Langlois, R. Maartens, and D. Wands, Phys. Lett. B 489, 259 (2000).

[6] S.W. Hawking, T. Hertog, and H.S. Reall, Phys. Rev. D 62, 043501 (2000); ibid., 63, 083504 (2001).

[7] D. S. Gorbunov, V. A. Rubakov, and S. M. Sibiryakov, JHEP 10, 15 (2001).

[8] A. Lewis, A. Challinor, and A. Lasenby, Astrophys. J. 538, 473 (2000).

[9] A. Lewis, PhD Thesis, University of Cambridge (2000).

[10] J.D. Barrow and R. Maartens, Phys. Lett. B 532, 153 (2002).

[11] K. Koyama and J. Soda, Phys. Rev. D 62, 123502 (2000); N. Deruelle, T. Dolezel, and J. Katz, Phys. Rev. D 63, 083513 (2001); P. Brax, C. van de Bruck, and A.C. Davis, JHEP 10, 026 (2001); M. Dorca and C. van de Bruck, Nucl. Phys. B605, 215 (2001); E.J. Copeland, A.R. Liddle, and J.E. Lidsey, Phys. Rev. D 64, 023509 (2001); A. Neronov and I. Sachs, Phys. Lett. B 513, 173 (2001); H. Kodama, A, Ishibashi and O. Seto, Phys. Rev. D, 62, 064022 (2000); C. van de Bruck, 
M. Dorca, R. Brandenberger, and A. Lukas, Phys. Rev. D, 62, 123515 (2000) D. Langlois, Phys. Rev. D, 62, 126012 (2000); D. Langlois, Phys. Rev. D, Phys. Rev. Lett. 86, 2212 (2000); H. Kodama, hep-th/0012132 C. Gordon and R. Maartens, Phys. Rev. D, 63, 044022 (2001); D. Langlois, R. Maartens, M. Sasaki and D. Wands, Phys. Rev. D, 63, 084009 (2001); O. Seto and H. Kodama, Phys. Rev. D 63, 123506 (2001); R. Maartens, gr-qc/0101059 (2001); H. Koyama and J. Soda, Phys. Rev. D 65, 023514 (2002); N. Sago, Y. Himemoto, and M. Sasaki, Phys. Rev. D 65, 024014 (2002); V. Sahni, M. Sami, T. Souradeep, Phys. Rev. D 65024014 (2002); A.R. Liddle and A.N. Taylor, Phys. Rev. D 65, 041301 (2002); H.A. Bridgman, K. Malik and D. Wands Phys. Rev. D 65, 043502; K. Koyama, Phys. Rev. D, to appear (gr-qc/0204047); G. Huey and J.E. Lidsey, astro-ph/0205236 (2002); S. Kanno and J. Soda, hep-th/0205188; C. Deffayet, hep-th/0205084 (2002); A. Riazuelo, F. Vernizzi, D. Steer, and R. Durrer, hep-th/0205220; D. Langlois, L. Sorbo, M. Rodriguez-Martinez, hep-th/0206146; G. Giudice, E. Kolb, J. Lesgourgues and A. Riotto, hep-ph/0207145; M. Bruni and P. Dunsby, hep-th/0207189; J. Bratt, A. Gault, R. Scherrer and T. Walker, Phys. Lett. B, to appear (astro-ph/0208133).

[12] B. Leong, P. Dunsby, A. Challinor, and A. Lasenby, Phys. Rev. D 65, 104012 (2002).

[13] A. Challinor, Class. Quantum Grav. 17, 871 (2000).

[14] P.K.S. Dunsby, B.A. Bassett, and G.F.R. Ellis, Class. Quantum Grav. 14, 1215 (1997).

[15] R. Maartens, Phys. Rev. D, 62, 084023 (2000);

[16] S. Mukohyama, Phys. Rev. D, 62, 084015 (2000);

[17] M. Sasaki, T. Shiromizu, and K. Maeda, Phys. Rev. D 62, 024008 (2000).

[18] S. Mukohyama, Phys. Rev. D 64, 064006 (2001).

[19] J. Soda and K. Koyama, hep-th/0205208 (2002).

[20] U. Seljak and M. Zaldarriaga, Astrophys. J. 469, 437 (1996).

[21] A.A. Starobinsky, Sov. Astron. Lett. 11, 3 (1985). 